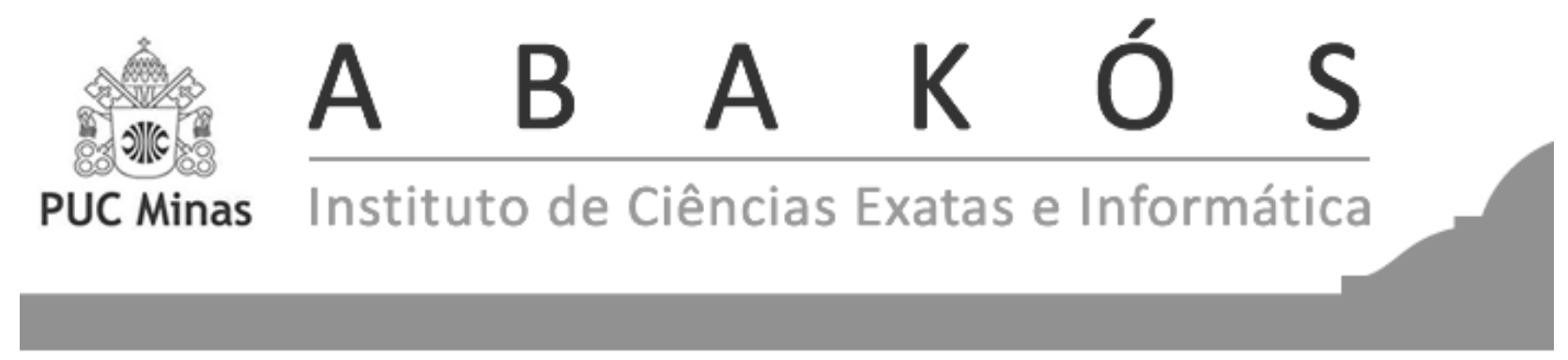

\title{
Desenvolvimento de uma Interface para Terapia de Biofeedback para Pacientes com AVC Utilizando Eletromiógrafo e Eletrogoniômetro*
}

\author{
Ana Caroline Pazeto ${ }^{1}$ \\ Larissa Teixeira de Oliveira ${ }^{2}$ \\ Raphael Diogo Teles ${ }^{3}$
}

\begin{abstract}
Resumo
O objetivo deste artigo é desenvolver uma interface que auxilie um tratamento por meio de uma terapia de biofeedback. Esta terapia envolve o retorno imediato de informações por meio de aparelhos sensoriais eletrônicos. Será desenvolvido um sistema para ser utilizado no tratamento de pacientes que tenham sofrido Acidente Vascular Cerebral (AVC). O AVC é um derrame cerebral, compromete a função neurológica das pessoas e é proveniente de um entupimento de vasos sanguíneos cerebrais. O programa criado é capaz de captar o sinal do eletromiógrafo e o sinal do eletrogoniômetro, comparando com um sinal de referência e identificando o quanto de força é aplicado na realização do movimento de extensão de braço. Utiliza-se, neste caso, a, abertura do ângulo a partir do envoltório do sinal. O sinal captado é relacionado com uma determinada imagem, obtendo, assim, um feedback visual na tela.
\end{abstract}

Palavras-chave: AVC. EMG. Eletrogoniômetro. Reabilitação. RMS. Biofeedback.

\footnotetext{
* Submetido em 24/06/2014 - Aceito 16/10/2014

${ }^{1}$ Engenheira Biomédica e Mestranda em Engenharia Clínica, Universidade Federal de Uberlândia. Faculdade de Engenharia Elétrica - Departamento de Engenharia BiomédicaBrasil- carolpazeto@gmail.com.

${ }^{2}$ Engenheira Biomédica e Pós graduanda em Engenharia de Segurança do Trabalho - PUC Minas, Universidade Federal de Uberlândia. Faculdade de Engenharia Elétrica - Departamento de Engenharia BiomédicaBrasil- larissateixeiradeoliveira@gmail.com.

${ }^{3}$ Engenheiro Biomédico, Universidade Federal de Uberlândia. Faculdade de Engenharia Elétrica - Departamento de Engenharia BiomédicaBrasil-raphaeldto@gmail.com.
} 


\begin{abstract}
The objective of this paper is to develop an interface that assists a treatment through a biofeedback therapy. This therapy involves the immediate return of information through electronic sensory apparatus. There will be developed a system to be used in the treatment of patients who have suffered Cerebral Vascular Accident (CVA). The CVA is a stroke, commits the neurological function of the people and is due a clogging of brain blood vessels. The generated program is able to pick up the signal of the EMG and the signal of the electrogoniometer, comparing with a reference signal and identifying how much force is applied in the implementation of the movement of arm extension. It is been used, in this case, the opening angle from the envelope of the signal. The received signal is related to a particular image, thus obtaining a visual feedback on the screen.
\end{abstract}

Keywords: AVC. EMG. Eletrogoniometry. Rehabilitation. RMS. Biofeedback. 


\section{INTRODUÇÃO}

O acidente vascular cerebral (AVC) é caracterizado pela rápida perda da função neurológica em decorrência de uma isquemia (entupimento) ou hemorragia (rompimento) de vasos sanguíneos cerebrais (NEUROINFORMAÇÃO, 2012; ROWE, 2001; PATOGÊNESE, 2002). Inicialmente, o paciente pode ter suas funções, como movimentação dos membros de determinado lado do corpo, comprometidas, além de apresentar dificuldades na elocução das palavras e ter seu campo visual prejudicado. Tal perda de capacidades corpóreas ocorre devido à destruição de células neurais (neurônios), provocada pela patologia - AVC.

A eletromiografia é uma técnica de monitoramento da atividade elétrica das membranas excitáveis do organismo, representando a medida dos potencias de ação do sarcolema, como efeito de voltagem em função do tempo. O sinal eletromiográfico (EMG) é o somatório algébrico de todos os sinais detectados em certa área, podendo ser afetado por propriedades musculares, anatômicas e fisiológicas, assim como pelo controle do sistema nervoso periférico e a instrumentação utilizada para a aquisição dos sinais (KANDEL, 2000).

Na Figura 1, observa-se um paciente que sofreu um AVC e, consequentemente, os traumas deixados pela doença, como por exemplo: o braço voltado para dentro, fletido e rodado, a mão fechada com os dedos escondidos na palma da mão, além da perna rodada para dentro, o pé, também, para dentro e o dedão para cima. Esta postura pós-AVC, classicamente chamada de postura de Wernicke-Mann, demonstra uma das sequelas mais importantes do AVC e de várias outras doenças como tumores cerebrais, traumas cranianos e esclerose múltipla, a espasticidade (NEUROINFORMAÇÃO, 2012).

\section{Figura 1 - Paciente acometido recentemente por um AVC com uma postura de Wernicke-Mann. (NEUROINFORMAÇÃO, 2012)}

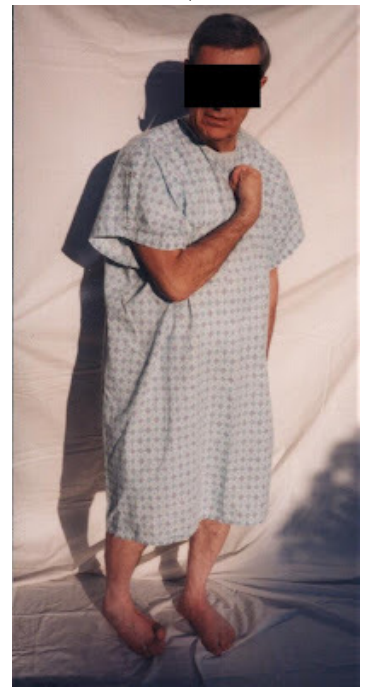

Com o intuito de contribuir com aqueles pacientes que tenham a postura WernickeMann, o objetivo deste artigo é medir a força que ele aplica para estender o braço por meio de um sinal biomédico, eletromiográfico, e então, observar o ângulo medido pelo sinal de um eletrogoniômetro. 


\section{REFERENCIAL TEÓRICO}

\subsection{Eletromiografia}

Atualmente, a eletromiografia tem sido introduzida como forma de avaliação em pacientes neurológicos. O EMG apresenta inúmeras aplicações na clínica médica para diagnóstico de doenças neuromusculares e na biomecânica no sentido de servir como um indicador de estresse muscular, indicador de padrões de movimentos e identificar parâmetros de controle do sistema nervoso. O EMG superficial tem se tornado muito valorizado como um sinal que permite avaliar a função do músculo, ajudando no tratamento fisioterápico (RAFII; HILLIS, 2006). O EMG tem sido utilizado em diversas áreas, entre elas reabilitação, ergonomia e diagnósticos neuromusculares.

O registro do EMG, portanto, é de suma importância, pois consegue analisar o músculo em diversas condições eletrofisiológicas: esforço, percentual de fibras rápidas e lentas, influências da temperatura corpórea, entre outros. Por oferecer um ótimo diagnóstico das contrações musculares, o registro do EMG vem aprimorando os estudos do movimento humano (FORTI, 2005; KANDEL, 2000).

Em tese, o EMG é um sinal biométrico proveniente da atividade elétrica da contração e relaxamento dos músculos.

\subsection{Fisiologia Muscular}

Os músculos são os tecidos do corpo humano responsáveis pelo movimento, uma vez que são capazes de aumentar ou diminuir o seu comprimento devido aos diversos estímulos musculares (KANDEL, 2000). O músculo é formado por um conjunto de fibras musculares, sendo que este é uma célula alongada e fina, a qual é constituída por sarcolema, sacorplasma e miofibrilas. No sacorplasma, encontram-se organelas, reticulo endoplasmático, túbulos T, mitocôndrias e miofilamentos chamadas também como miofribilas (KANDEL, 2000).

As miofibrilas são dividas em actina e miosina. O sarcômero é a unidade contrátil muscular e é formada pela repetição de actinas e miosinas. Portanto, as miofibrilas é que são as responsáveis pela contração muscular (GUYTON; HALL, 1997).

As fibras musculares têm sua inervação feita por neurônios do sistema nervoso periférico, os motoneurônios inferiores (KANDEL, 2000).

A unidade motora é constituída de axônio, neurônio motor e sistema nervoso. Cada músculo tem uma quantidade de unidade motora relacionada, e este número é chamado de taxa 
de inervação. As unidades motoras são classificadas de acordo com a atividade responsável de cada músculo. Por exemplo, se um músculo é responsável por movimentos e ajustes finos, tem um número menor de fibras musculares por unidade motora. E, quando o músculo produz força, ele é composto por unidades motoras maiores, com alta taxa de inervação, com centenas de fibras musculares inervadas por uma única fibra nervosa (MARCHETTI; DUARTE, 2006).

A junção neuromuscular ou placa motora é a região em que há contato entre a terminação nervosa e as fibras musculares; é, normalmente, localizada na região mediana da fibra muscular (KANDEL, 2000; KREBS, 1993).

\subsection{Eletrogoniômetro}

A goniometria é uma técnica para medir ângulos articulares, que possibilita uma avaliação da amplitude articular, descrição e compreensão dos movimentos realizados por segmentos adjacentes, proporcionando uma análise para controle de reabilitação (BONTRAGGER, 1999). Os eletrogoniômetros são uma versão eletrônica do goniômetro manual, possibilitando assim uma mensuração dinâmica do deslocamento angular.

A eletrogoniometria é uma variação do método e permite, com o uso de eletrogoniômetro, mensurar, eletronicamente, a variação angular das articulações durante todo o movimento (KREBS, 1993). O eletrogoniômetro é um instrumento que, ao ser estimulado na mudança da amplitude do movimento articular, gera sinais elétricos que são tratados e transformados em dados numéricos que quantificam o deslocamento angular. A resposta é, então, levada à tela do computador (ROWE, 2001).

A posição de alocação do instrumento é na parte proximal e distal da articulação que vai ser medida (BONTRAGGER, 1999).

\subsection{Acidente Vascular Cerebral}

O acidente Vascular cerebral é uma interrupção súbita no fluxo sanguíneo do encéfalo, causando tanto obstrução de uma artéria, AVC isquêmico, quanto uma ruptura arterial, AVC hemorrágico. A patologia é, portanto, uma alteração na circulação cerebral ocasionando um deficit transitório ou definitivo no funcionamento de uma ou mais partes do cérebro, resultando em perda de função neurológica (COLLINS, 1998; MARCHETTI; DUARTE, 2006).

O AVC representa a terceira causa de morte ao redor do mundo e uma das principais causas de incapacidade física e mental. A sua prevalência é alta e, atualmente, 90\% dos sobreviventes desenvolvem algum tipo de deficiência. Dentre as manifestações clínicas, podemos 
citar os prejuízos das funções sensitivas, motoras, de equilíbrio e de marcha, além do deficit cognitivo e de linguagem (INGALL, 2004).

Atualmente, uma das formas mais encontradas para a avaliação de um paciente é por meio da utilização de escalas. Elas traduzem uma informação clínica para uma linguagem objetiva e universal, proporcionando uma base científica para a comunicação. As capacidades motoras podem ser quantificadas por escalas e limiares. Esses instrumentos pontuam as atividades que podem ser reavaliadas, oferecendo ao terapeuta a condição de analisar a eficiência do processo terapêutico (PATOGÊNESE, 2002).

A incapacidade funcional é um dos outcomes mais importantes após um AVC, sendo a sua avaliação uma das mais complexas, pois envolve a conjugação de vários fatores. A fisioterapia tem, portanto, um papel fundamental no processo de reabilitação, sendo que grande parte da recuperação funcional pode ser atribuída a sua prática efetiva.

Estudos sobre a reabilitação em pacientes que apresentam sequelas decorrentes de AVC têm comprovado, cada vez mais, a urgência de que o tratamento reabilitador seja iniciado o mais breve possível, ainda dentro da Unidade de Tratamento Intensivo, ou logo após a estabilidade clínica (GARCÍA, 1994; KANDEL, 2000).

\section{METODOLOGIA}

Esta pesquisa foi realizada na Universidade Federal de Uberlândia (UFU). O projeto consiste do desenvolvimento de uma interface no ambiente de trabalho Labview, responsável pela visualização do sinal e do biofeedback. O sinal é captado por meio do kit MIOSYSTEM, apresentado na Figura 3, que fica localizado no laboratório de Engenharia Biomédica (BIOLAB) da UFU e retorna frames de imagens como biofeedback.

A Figura 2 mostra o diagrama de blocos do sistema.

Figura 2 - Diagrama de blocos.
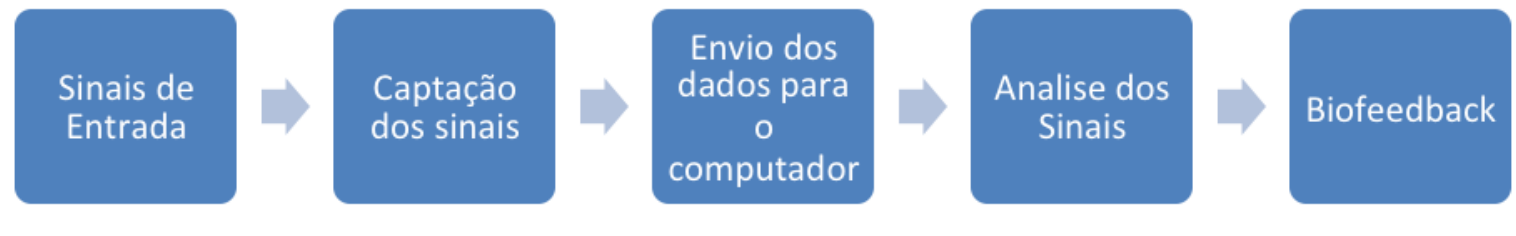

A seguir os materiais utilizados para esta pesquisa.

O hardware utilizado para captação é o kit disponível no BIOLAB, no qual se utiliza o módulo de eletromiografia e eletrogoniômetro para captação dos sinais. O kit já vem como os dispositivos para medição do EMG e do eletrogoniômetro. 
Figura 3 - Kit MIOSYSTEM

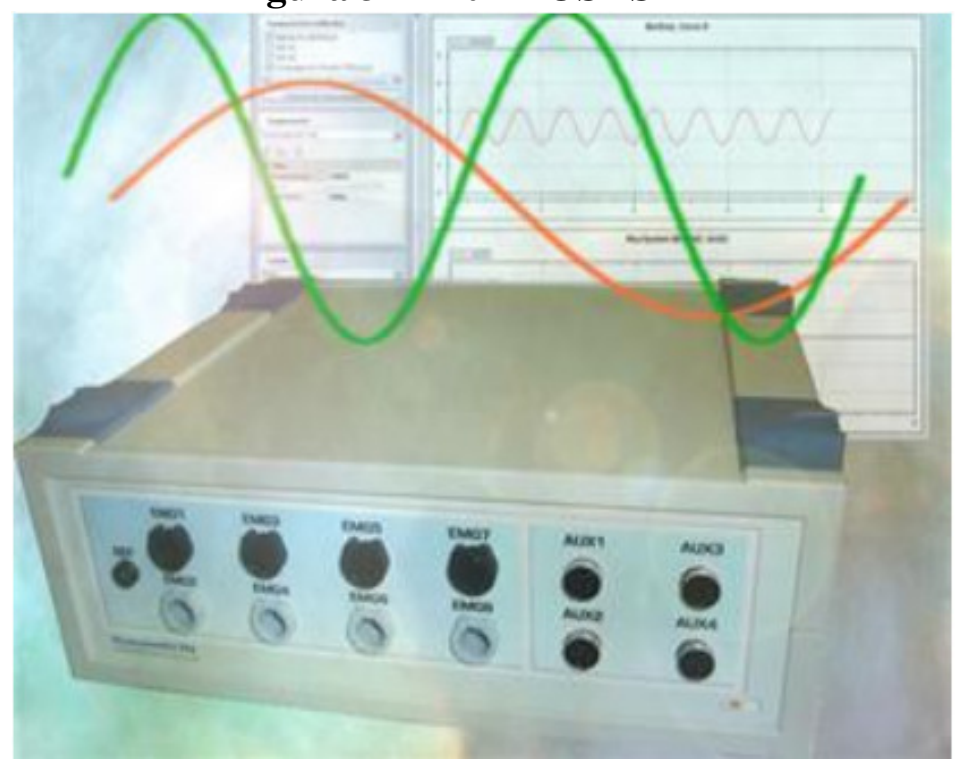

O módulo de EMG utilizado é o conector para eletrodo ativo, posicionado no canal 4.

O módulo do eletrogoniômetro é colocado no canal 1 do kit.

O eletrodo escolhido para este projeto foi o eletrodo de Cloreto de Prata ( $\mathrm{AgCl})$. Antes de colocar o eletrodo, há a necessidade de realizar uma limpeza da pele, para reduzir a sua impedância. Não há necessidade de aplicar gel, já que este eletrodo já vem com um gel condutor adicionado a ele.

Os eletrodos são posicionados nos músculos flexores e extensores do braço, mais precisamente o pronador redondo e o braquirradial, que são responsáveis pela flexão, e o ancôneo, que é responsável pela extensão.

O kit MIOSYSTEM disponibiliza uma comunicação universal serial bus (USB) com o computador. Esta é usada para enviar os dados captados pelos módulos para o computador. O programa utilizado para comunicar os sinais enviados é o Instacal, uma placa de aquisicionamento virtual, que comunica tanto com o kit quanto com o Labview. A board \#1 foi empregada para aquisição do sinal do kit. Estão disponíveis oito canais signal ended, porém, só são utilizados os canais 0 e o 3, um referente ao eletrogoniômetro e o outro ao EMG, respectivamente.

No software LABVIEW existem programas que são exemplos do funcionamento de algumas funções, que foram utilizadas como exemplos base para o trabalho, como foi o 'Xaincnbg'. Ele comunica-se com o Instacal que manda o sinal captado pelo kit do Biolab.

Por meio desse exemplo, podem ser escolhidos os canais com os quais se deseja trabalhar. Utiliza-se este no modo Singleio, com um range de -/+10V (Volts) com 3 canais, já que os canais que utilizamos é o 0 e o 3. 


\section{EXPERIMENTO}

O primeiro passo foi separar os sinais.

A Figura 4 mostra a utilização do bloco Slip Signal para separar os sinais. Os sinais utilizados estão no canal 0 e no canal 3. A Figura 5 mostra o bloco Slip Signal.

\section{Figura 4 - Aquisição do Sinal e separação dos sinais}

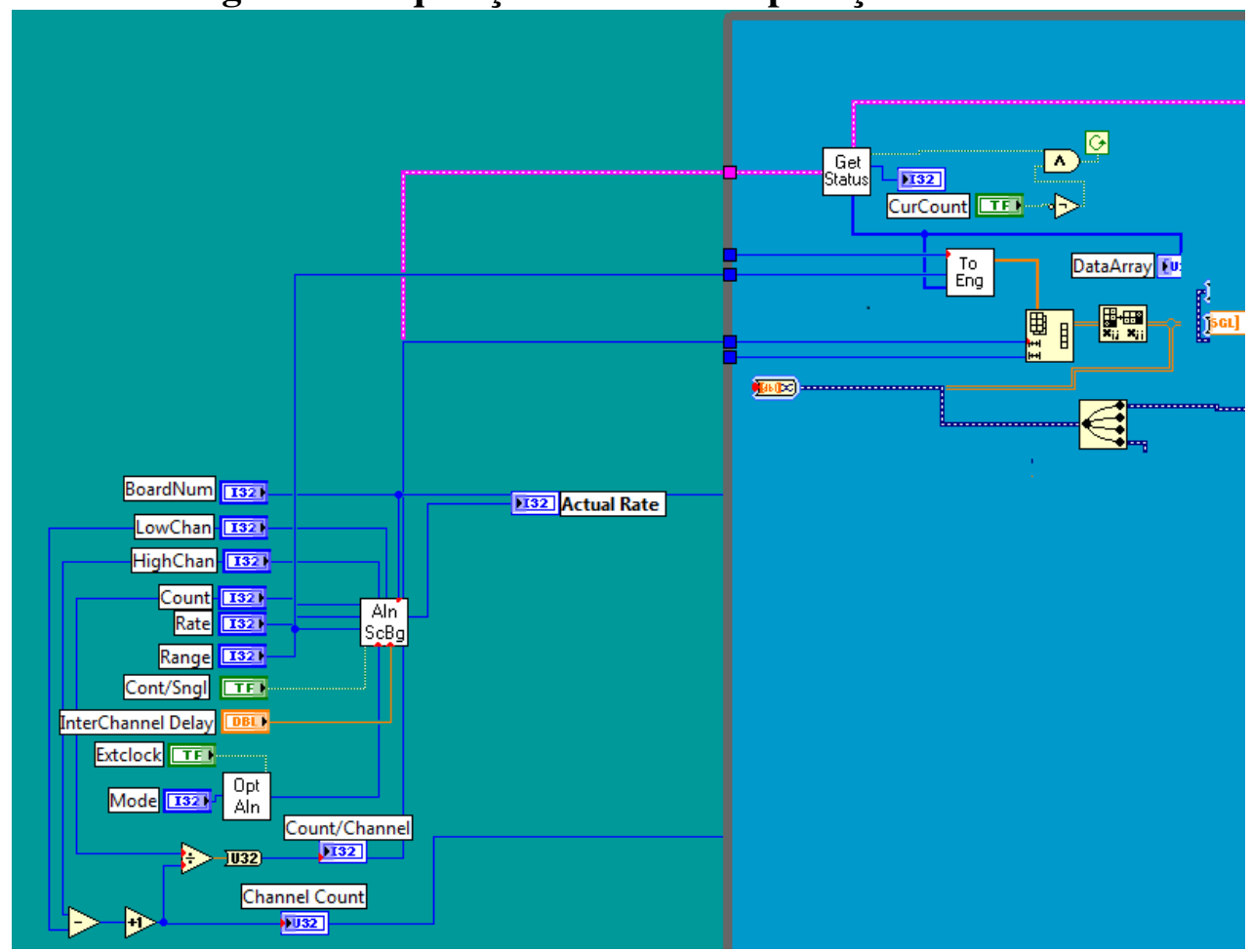

Figura 5 - Função Slip Signal

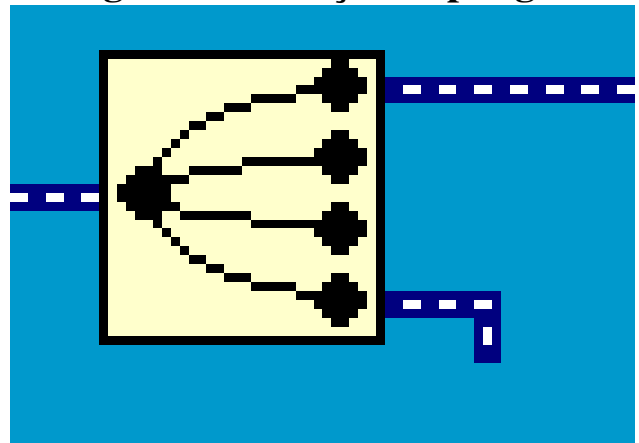

Em seguida, processa-se o sinal captado e, então, o processamento do sinal biomédico pode ser feito de várias maneiras. Neste caso, foi utilizado o envoltório do sinal, e a partir dele, foi calculada a média dos valores.

O envoltório linear é um tipo de média móvel que indica a magnitude do sinal. O root mean square (RMS) é uma medida estatística da magnitude de uma quantidade variável. Foi utilizada tal medida para captar a magnitude do sinal e comparar os dois sinais por este valor e, assim, saber a intensidade de força que está sendo aplicada pelo paciente para realizar determinado movimento. 
O bloco no Labview para o cálculo do RMS é a função RMS (DBL), mostrado na Figura 6. O cálculo do RMS no Labview é feito pela fórmula (1):

$$
\psi_{x}=\sqrt{\frac{1}{n} \sum_{i=0}^{n-1}\left|x_{i}\right|^{2}}
$$

onde $\psi$ é o valor final do RMS calculado e $n$ é o número de elementos do vetor X.

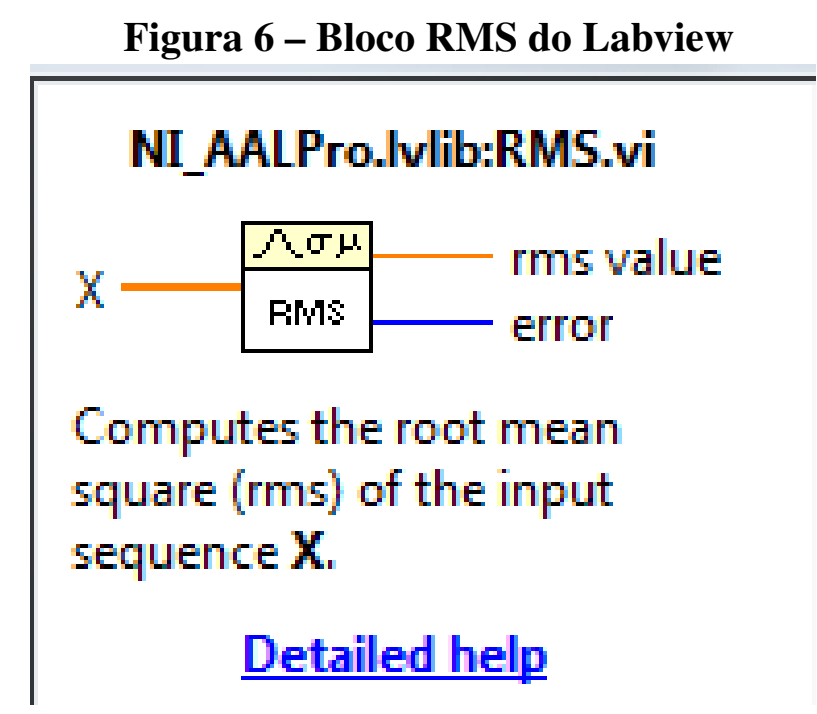

Para cada sinal, é calculado o RMS e registrado em uma caixa indicadora. O RMS é comparado com um limiar. Este limiar pode variar e pode ser alterado pelo usuário dependendo do nível de cada paciente. Posteriormente comparado com um limiar, os dois sinais RMS são analisados, e assim, são encontrados os dados necessários para o estudo. Se a comparação com um limiar for verdadeira entre um case e outro, uma imagem de feedback é apresentada. Observa-se, na Figura 7, como é feita a comparação.

\section{Figura 7 - Comparação dos limiares e os cases}

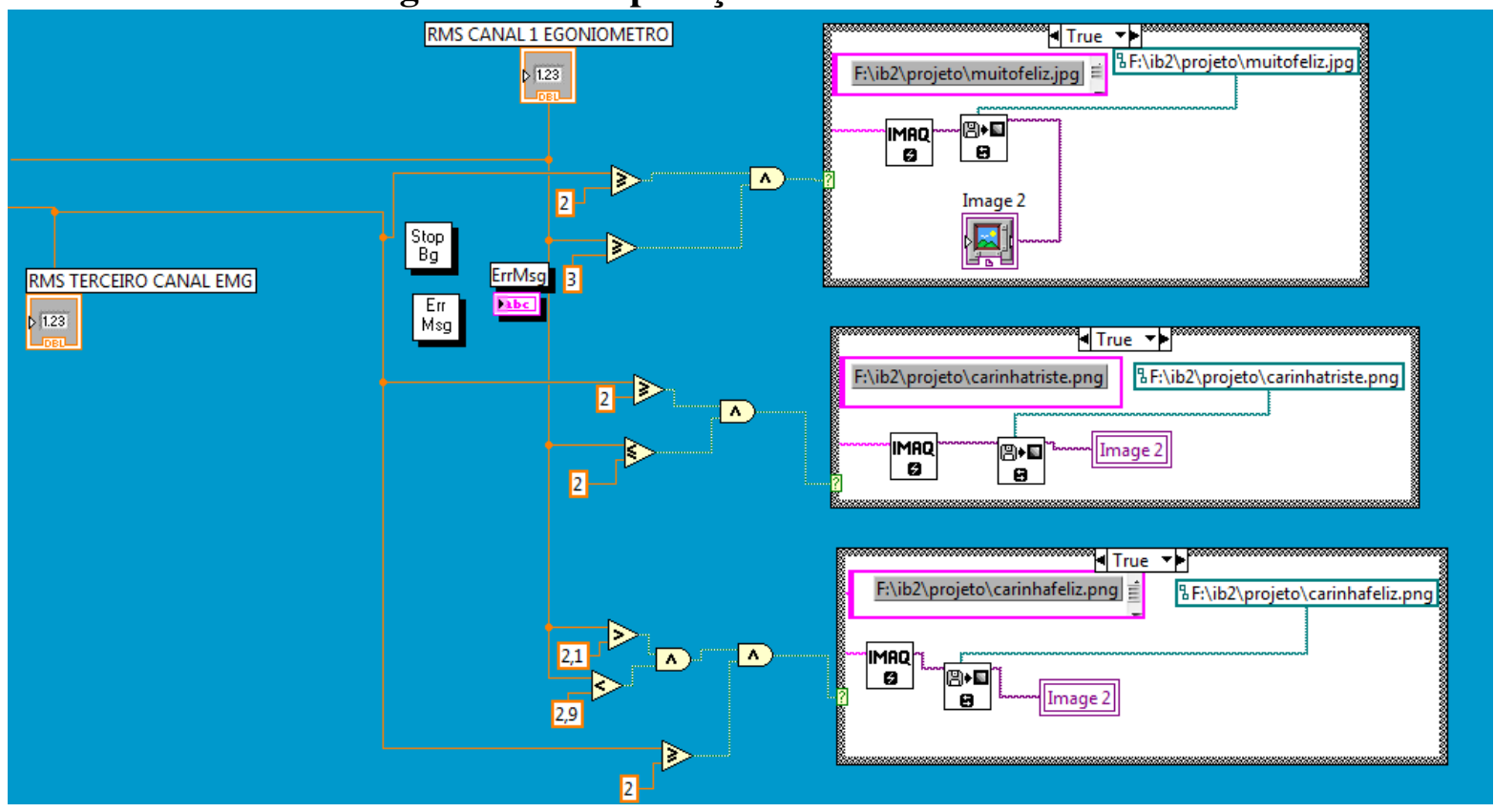


O biofeedback é feito por meio de frames de imagens. A primeira imagem, apresentada na Figura 8, que é a de uma "carinha" triste, mostra que o ângulo atingido pelo movimento, resultante da força aplicada, não foi muito grande.

\section{Figura 8 - Primeira imagem, nível 1}

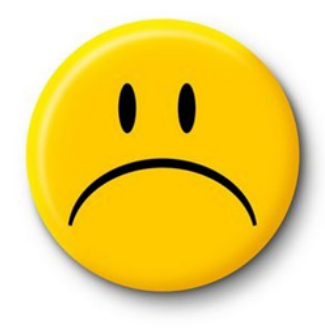

A segunda imagem, ilustrada pela Figura 9, é uma "carinha" feliz, mostra que a contração muscular conseguiu a abertura de um ângulo mediano.

\section{Figura 9 - Segunda imagem, nível 2}

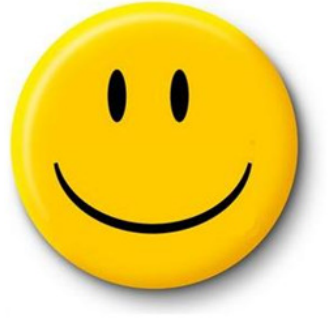

E, por fim, a última imagem, apresentada na Figura 10, é uma "carinha" muito feliz que mostra que o paciente alcançou o objetivo, que é abrir um ângulo maior com sua força.

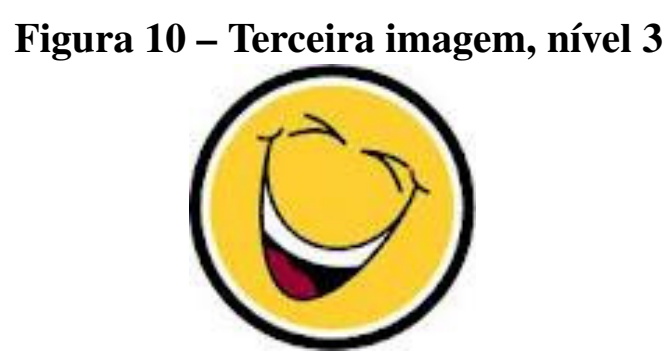

\section{RESULTADOS}

Para verificação do software, realizaram-se alguns testes. Primeiramente, foram realizados testes para a escolha de um limiar, já que o mesmo pode variar de paciente para paciente. Este limiar é ajustado pelo especialista da área, pelo fato de que um paciente que acabou de iniciar o tratamento pode ter uma força muscular pequena e, para não desestimulá-lo, o limiar pode ser aumentado gradativamente. Essa é uma informação importante para o usuário acompanhar a evolução do paciente.

Para esse teste, foram realizados experimentos para escolha do limiar somente para validar o processo. Escolheu-se o limiar tanto do EMG quanto do eletrogoniômetro. Para o EMG, definiu-se que o RMS deveria ser maior que 2. No eletrogoniômetro, o RMS variou de 0 a 4. 
O limiar utilizado para a primeira imagem foi um RMS do eletrogoniômetro menor que 2 , já que pelos testes, foi visto que esse foi um ângulo muito pequeno. Para a segunda imagem, o limiar do RMS é entre 2 e 3. E para a ultima imagem, o limiar é maior que 3. O sistema funcionou de acordo com os limiares e gerou um feedback.

Observa-se, também, a importância de se ter disponível um equipamento em tempo real para que se possa perceber o avanço do tratamento do paciente e se tenha um total aproveitamento da interface.

\section{DISCUSSÃO}

Inicialmente, foram identificadas algumas dificuldades para adaptação com o Labview, pois era um ambiente de trabalho novo e com um tipo de linguagem diferente do usual. Nele, a programação é feita por meio de blocos e a equipe estava acostumada a trabalhar com linhas de código de programação.

Outra dificuldade foi relativa à identificação do problema e da busca de sua solução. Com o desenvolvimento do projeto e o maior aprofundamento de conhecimentos, tanto da interface quanto das dificuldades dos fisioterapeutas, as dificuldades foram gradativamente superadas.

Nota-se, assim, que este trabalho foi útil para que a equipe pudesse aprender uma nova linguagem, além de adquirir conhecimento para a escolha de todos os materiais a serem utilizados. Espera-se que esse trabalho apresente uma significativa contribuição para o campo das ciências médicas.

\section{CONCLUSÃO}

O projeto alcançou seu objetivo, que era, por meio do sinal de EMG e do eletrogoniômetro, realizar um biofeedback para pessoas que tenham sofrido de AVC.

Apesar de não terem sido realizados testes com pessoas que tenham o acidente, acreditase que a proposta apresentada venha auxiliar o tratamento destes pacientes.

O próximo passo deste trabalho é a realização de testes em pacientes com AVC para validar o sistema integralmente. 


\section{Referências}

BONTRAGGER, EL. Instrumented gait analysis sistems. In: DELISA JA. GAIT ANALYSIS IN THESCIENCE OF REHABILITATION. Washington, 1999. Disponível em: <http://www.vard.org/mono/gait/bontrager.htm/> Acesso em: 20 set. 2014.

COLLINS, Robert C. Neurologia. Rio de Janeiro: Guanabara Koogan, 1998.

ENOKA, R. M. Bases neuromecânicas da cinesiologia. São Paulo: Manole, 2000.

FORTI, F. Análise do sinal eletromiografico em diferentes posicionamentos, tipos de eletrodos, angulos articulares e intensidades de contração. 2005. Dissertação (Mestrado) Universidade Metodista de Piracicaba, Programa de Mestrado em Engenharia Biomédica, Piracicaba.

GARCÍA, M. T. Florez. Scalas de valoración funcional: aplicaciones, caraterísticas y criterios de selección. Rehabilitación, v. 6, n. 28, p. 373-6, 1994.

GUYTON, C.; HALL, J. E. Tratado de Fisiologia Médica. 9. ed. Rio De Janeiro: Guanabara Koogan, 1997.

INGALL, Timothy. Stroke-incidence, mortality, morbidity and risk. Journal of insurance medicine (New York, N.Y.), v. 36, n. 2, p. 143-152, 2004. Disponível em: <http://apt.allenpress.com/aptonline/?request=get-document\&issn=0743$6661 \&$ volume $=036 \&$ issue $=02 \&$ page $=0143>$.

KANDEL, E. R. Fundamentos da Neurociência e do Comportamento. Rio de Janeiro: Guanabara Koogan, 2000.

KREBS, D. E. Biofeedback. In: O‘SULLIVAN, S. B., SCHIMITZ, T. J. Fisioterapia: avaliação e tratamento. 2. ed. São Paulo: Manole, 1993.

MARCHETTI, P. H.; DUARTE, M. Instrumentação em eletromiografia. São Paulo: Universidade de São Paulo, 2006.

NEUROINFORMAÇÃO. O uso de toxina botulínica após um AVC. 2012. Disponível em: <http://neuroinformacao.blogspot.com.br/2012/02/o-uso-de-toxina-botulinica-aposum-avc.html> Acesso em: 23 Abr. 2012.

PATOGÊNESE, Lewis S. R. Classificação e epidemiologia das doenças vasculares cerebrais. In: L. P. ROWALAND. Merrit. Tratado de Neurologia. 10. ed. Rio de Janeiro: Guanabara Koogan, 2002. p. 184-95.

RAFII, M. S.; HILLIS, A. E. Compendium of cerebrovascu-lar diseases. Int Rev Psychiatry, v. 18, n. 5, p. 395-407, 2006.

ROWE, P. J. Movimento funcional humano. In: DURWARD, B. R., BAER, G. D. Movimento funcional Humano. São Paulo: Manole, 2001.

SILVA, J. M.; LIMA, M. O.; JÚNIOR, A. R. P. Efeito agudo da estimulação vibratória em hemiparéticos espáticos pós-acidente vascular encefálico. Revista brasileira de engenharia biomédica, v. 27, n. 4, p. 225-230, 2011.

WEBSTER, J. G. Medical Instrumentation: Application and Design. USA: John Wiley \& Sons, 2005. 